\title{
Vestibular involvement in a passive transport and return task
}

\author{
SUSAN MILLER \\ Teachers College, Columbia University, New York, New York \\ MICHAEL POTEGAL \\ New York State Psychiatric Institute, New York, New York
}

and

LARRY ABRAHAM

University of Texas, Austin, Texas

\begin{abstract}
Rats were trained to return to a water spout after being passively transported away from it along paths containing a 90-deg turn. Path lengths were successively increased to as much as $132 \mathrm{~cm}$ by a modified titration procedure. The task was successfully relearned after enucleation. Subsequent vestibular nucleus damage produced a severe, lasting deficit in this task when compared with the effects of cerebellar cortex lesions. In contrast, the vestibular lesions produced only a mild, transient impairment in an olfactory localization task. The most anterior vestibular lesions also affected air righting.
\end{abstract}

The hypothesis that vestibular information might be important for spatial orientation has long had intuitive appeal (Barlow, 1964; Exner, 1893; Watson, 1907; see Potegal, 1982, for a recent review). The vestibular apparatus supplies information about linear acceleration and, in the range of normal movement, angular velocity (Jones \& Milsum, 1970), which could conceivably be used to guide navigation through space. To date, however, only limited evidence has been offered in support of this hypothesis. For example, in nine studies performed between 1893 and 1951, evidence for vestibular involvement in bird migration was sought; the results were systematically negative (see Barlow, 1964, for review). Barlow has pointed out that vestibularly dependent navigation is based on an inertial guidance system. Such systems tend to accumulate errors; the longer the distance, the greater will be the net error. Thus, a vestibularly dependent system might be most useful for navigating short distances, but could be only supplementary in long-distance bird migration.

In contrast to bird navigation, laboratory mazetype tasks would seem to be a more appropriate situation for detecting a vestibular contribution to spatial orientation. Indeed, from the earliest maze-type studies to the present, authors have speculated about and argued for a vestibular contri-

We would like to thank John Nee for his help in preparing the computer simulations and Joe Beattus and Richard Greenstein for their help in testing the rats. Requests for reprints may be sent to: Michael Potegal, New York State Psychiatric Institute, 722 West 168th Street, New York, New York 10032. bution (e.g., Watson, 1907; Zoladek \& Roberts, 1978). Most of these arguments are based on indirect evidence. That is, if an animal's maze performance persists after cues in the obvious sensory modalities have been eliminated or made irrelevant, it is suggested that the performance may be guided by vestibular input. This line of argument was, for example, the basis of Dashiell's claim $(1930,1959)$ for a vestibularly guided "direction orientation," by which he meant that a rat could weave his way through a series of barriers without losing track of the direction in which he was heading. In this mode, the vestibular system is being used to detect forced angular deviations from the rat's original orientation. The same sort of argument has also been used to support the claim that vestibular input may be operated upon in a more sophisticated spatial mode. According to this hypothesis, the organism determines from its vestibular feedback the distance and direction traveled from some starting point and incorporates this information into a spatial map. The empirical evidence offered is that, in at least some studies, animals have produced shortcuts and detours, indicating the use of spatial mapping in the absence of obvious intra- or extramaze cues (Honzik, 1933; Wolfle, 1935).

One of the problems with this sort of reasoning is that the active locomotion produced by animals while learning and performing in most maze studies necessarily generates kinesthetic (joint sense) and proprioceptive (muscle sense) feedback in addition to that coming from vestibular endorgans. These other sources of information might therefore be 
the basis of "direction orientation" and/or shortcut and detour ability. One way to eliminate taskrelevant kinesthetic and proprioceptive feedback while at least partially maintaining the vestibular input is through passive transport. When an animal is transported along a path in a small, enclosed vehicle, it can detect angular deviations in the path with the cristae of its semicircular canals, although active locomotion and the concomitant kinesthetic and proprioceptive feedback have obviously been precluded. Furthermore, detection of the linear accelerations occurring during the journey, when combined with suitable timekeeping, can yield taskrelevant information about linear displacements.

The Russian investigator Beritoff (1965) is among the few to have extensively studied animals' performance under these conditions. For example, he has reported that if a blindfolded cat or dog is passively transported along a path it can, when released, return directly to its starting point to obtain a food reward. Unfortunately, he did not publish the details of the training procedures for his "return from passive transport"' (RPT) tasks.

Even under the conditions of passive transport, however, an animal need not necessarily rely on vestibular input for spatial orientation. Various other sensory modalities may still be used; these could range from subtle cues in the obvious modalities which were overlooked by the experimenter to more exotic possibilities such as echolocation (Riley \& Rosenzweig, 1957) or a "magnetic sense" (Walcott, Gould, \& Kirschvink, 1979). Thus, it is important to have evidence going beyond indirect arguments. One kind of direct evidence would be the demonstration that destruction of vestibular input specifically eliminated RPT performance. Beritoff (1965) has, in fact, reported that labyrinthectomy would do just this.

In an effort to develop a test which could be used to isolate vestibular contributions to spatial orientation in rats, we devised training procedures for a RPT task comparable to Beritoff's. We then found that this RPT performance was not dependent upon visual cues. Finally, we discovered that performance of a RPT task was grossly impaired following vestibular nuclear damage, while performance in another spatial localization task was not.

\section{EXPERIMENT 1}

\section{Method}

\section{Subjects}

Experimentally naive, male Long-Evans hooded rats (Blue Spruce Farms, Altamont, N.Y.), weighing 300-325 g, were individually housed and given ad-lib lab chow. Thirty rats started in Experiment 1; however, data will be reported for only the 13 animals that finished all three of the following experiments.

\section{Apparatus}

RPT training took place in a visually homogeneous, $2.3 \times$ $2.3 \mathrm{~m}$ testing room. Eight identical water bottles fitted with stopcocks were mounted on vertical stands supported by two $30-\mathrm{cm}$ wooden arms. The bottles were placed in each corner and the center of each wall (see Figure 1). Each bottle served as a target bottle at least once in each session. The rat was transported in an enclosed wheeled vehicle, $20 \times 17 \times 10 \mathrm{~cm}$. When opened, the vehicle's back panel served as a ramp to the floor (Figure 1c); when closed, a small opening in the panel allowed the rat to drink from the spout that protruded $5 \mathrm{~mm}$ into the vehicle (Figure 1b).

\section{Pretraining}

Rats were handled for $10 \mathrm{~min} /$ day on Days 1 through 9 and habituated to being in the vehicle for $3 \mathrm{~min} /$ day on Days 3 through 5. Beginning on Day 6 and throughout the remainder of the experiment, they were chronically water deprived: water was available in the home cage for $20 \mathrm{~min} /$ day following the day's training. On Days 6 through 9, the rats were habituated to drinking in the vehicle. Shaping to the RPT task occurred in four stages, beginning on Day 10. In the first stage, the target bottle and vehicle were enclosed within a $70-\mathrm{cm}$-long transparent Plexiglas training guide which allowed vision of, but not access to, the inoperative bottles (see Figure 1c). The rat was allowed to drink briefly from the water spout protruding into the vehicle. Then the vehicle was pulled $15 \mathrm{~cm}$ from the bottle, and the rat was released by lowering the back panel. The rats were given repeated daily trials in returning to (and drinking from) the target bottle until they had done so four times within one of the daily 10-min

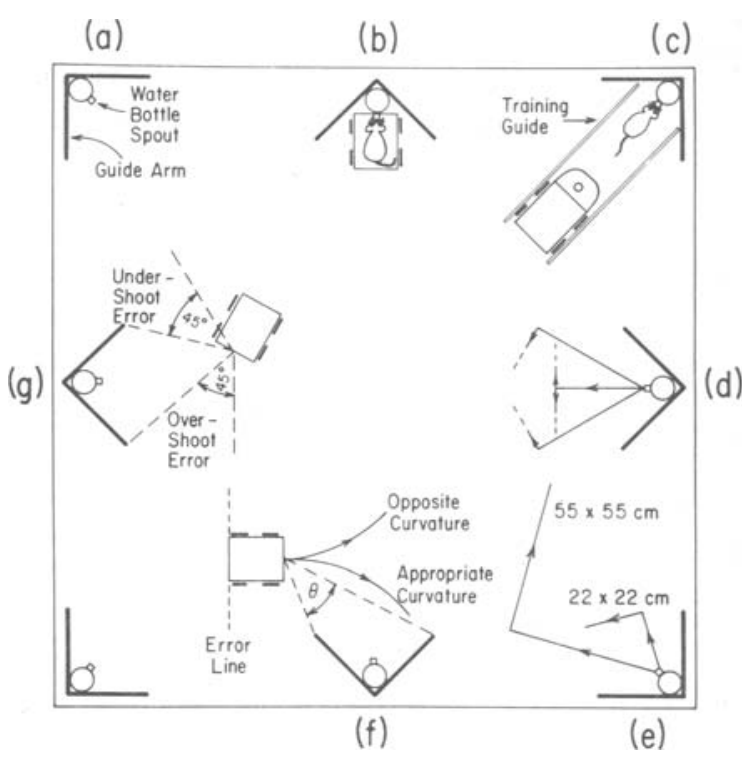

Figure 1. (a) Plan view of water-bottle/guide-arm assembly. Note that the water bottle is not drawn to scale. (b) Rat drinking from water-bottle spout protruding into vehicle. (c) Rat drinking from spout after having been released from vehicle within training guide. (d) The four possible path configurations from a midwall bottle. (The second leg of each path has been left incomplete for clarity of representation.) (e) Sample $22 \times$ $22(=44) \mathrm{cm}$ and $55 \times 55(=110) \mathrm{cm}$ corner bottle paths. (f) Illustration of chance success angle $(=\theta)$, examples of (1) appropriate and opposite trajectory curvature and (2) the "error line" beyond which rats were not allowed to wander. (g) Forty-fivedegree sectors adjacent to guide arms which defined undershoot and overshoot errors. 
sessions. In Stages 2 through 4 and throughout the remainder of the experiment, a modified path-length titration procedure was used. The rat was initially drawn $11 \mathrm{~cm}$ from the bottle and released. A trial was successful if the rat returned to the target bottle within $30 \mathrm{sec}$. After three consecutive successes (criterion performance), the path was increased by $11 \mathrm{~cm}$. If the rat failed to return within $30 \mathrm{sec}$, it was picked up and placed in the vehicle. When such an error occurred, the path length was subsequently reduced by $11 \mathrm{~cm}$. Following completion of one successful trial after one or more consecutive errors, the path length was increased to the length it had been prior to the error(s). In Pretraining Stage 2 , these procedures were carried out within the training guide until the rat reached criterion at a distance of $44 \mathrm{~cm}$. In Stage 3, this procedure was repeated without the training guide and with only a single bottle present in the room. The experimenter stood behind the vehicle while releasing the rat and recording its responses. Ten daily trials were given until criterion performance at $55 \mathrm{~cm}$ was reached. As above, an error was defined as a failure to return to the target bottle in $30 \mathrm{sec}$. In addition, an error was scored if all four of the rat's paws crossed the 180 -deg line (i.e., if it started to go behind the vehicle; see Figure 1f). To reduce the interference with training caused by the rats' exploratory tendencies, several rats were simultaneously allowed to freely explore the testing room for $10 \mathrm{~min}$ immediately prior to their daily training. Of course, water was not available from the bottles during this period. Stage 4 pretraining was a repetition of that of Stage 3, except that all eight bottles were present. Again, an error was scored if the rat reached an incorrect bottle within $30 \mathrm{sec}$ after release.

\section{Training}

Training began with the introduction of paths containing a left or right 90-deg turn (Figures 1d and 1e). The rotations at the vertex of the angle were briskly executed. Each daily session consisted of eight angle and two linear paths. The linear paths were interpolated on Trials 3,4 , or 5 and 7,8 , or 9. Criterion performance and path-length titration rules for angle paths were as above. Path lengths were incremented (initial segment first) as follows: $22 \times 22,33 \times 22,33 \times 33, \ldots, 66 \times 55,66 \times 66 \mathrm{~cm}$. (In all figures and tables, only the total length of each angular path will be given; e.g., $22 \times 22=44,66 \times 55=121$.) Errors were defined as leaving the vehicle and (1) going behind the vehicle (Figure 1f) or (2) reaching an incorrect bottle within $30 \mathrm{sec}$. Failure to leave the vehicle within $30 \mathrm{sec}$ constituted a balk; balks did not count as trials or errors and did not affect subsequent path length. However, if a rat committed either two consecutive

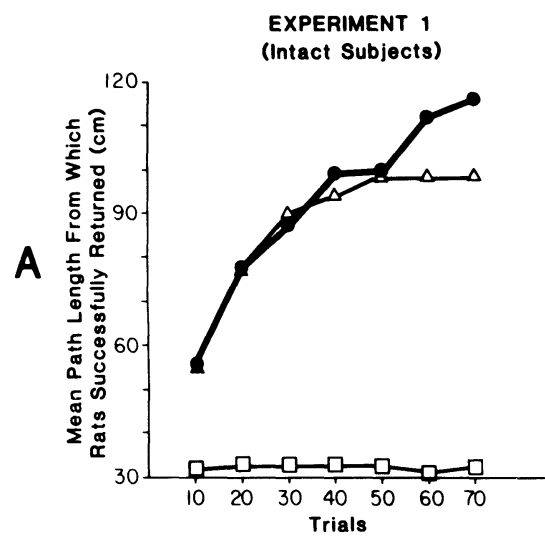

EXPERIMENT 2

(After Enucleation)
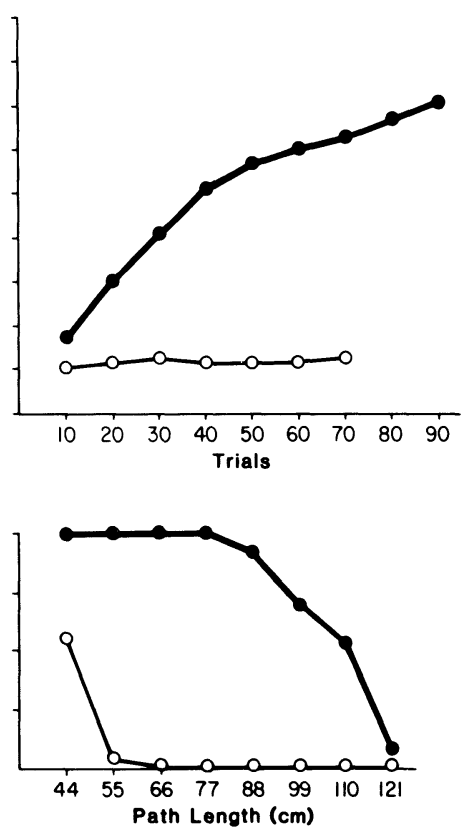

EXPERIMENT 3

(After Brain Lesions)
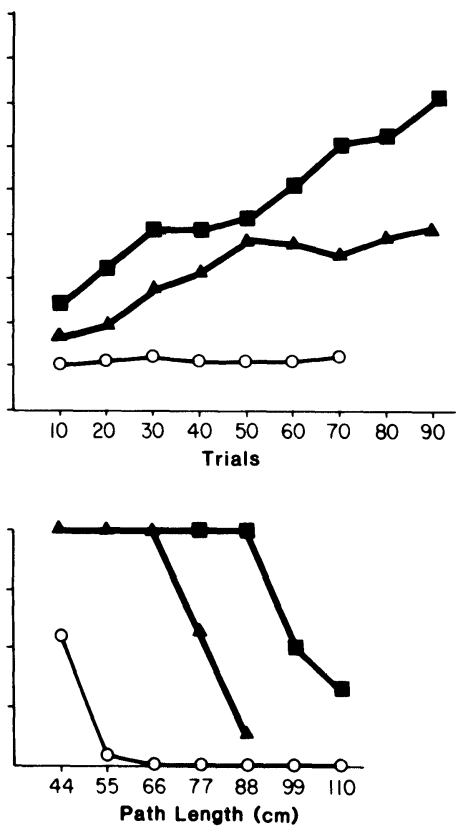
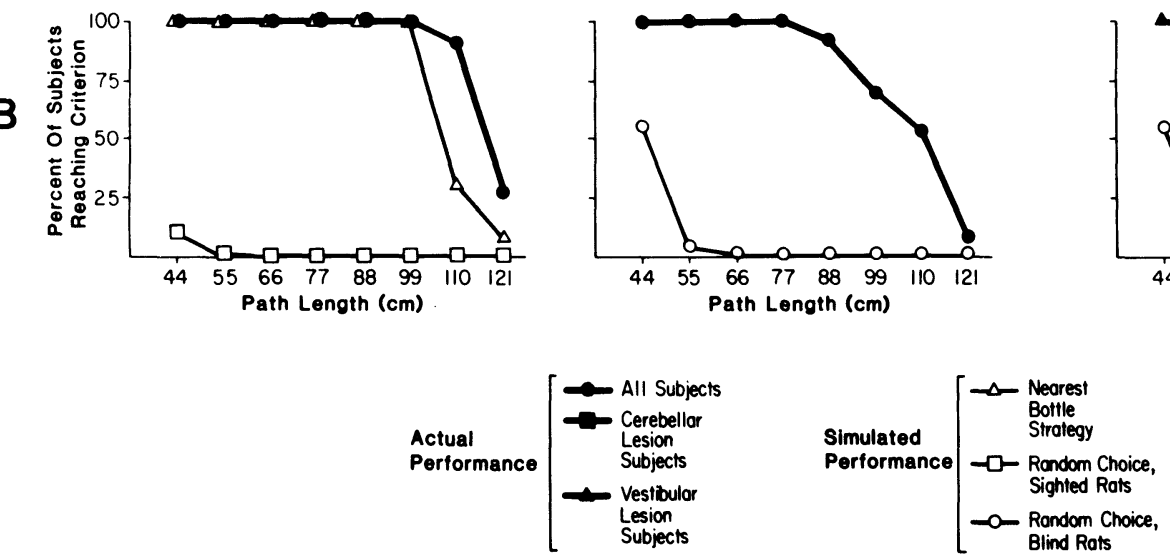

Figure 2. (A) The mean cumulative path length per each 16-trial block from which rats successfully returned divided by the number of successful trials in that block. Simulations were carried out only to 70 trials. The same simulation of random performance was used in Experiments 2 and 3. Note that in Experiment 1 actual performance at the longest path length cannot be explained by the "nearest-bottle" strategy. One or two animals in Experiments 2 and 3 finished only 84 to 86 of the 90 planned trials; their expected performance on the missing trials was extrapolated from their performance on the first trials of the last block. (B) Percent of subjects reaching criterion at successive path lengths. These percentages were generated on the basis of the first 70 trials for each rat in Experiment 1, 86 trials in Experiment 2, and 84 trials in Experiment 3. The same simulation of random performance was used in Experiments 2 and 3. 
errors or balks, it was "timed out" (i.e., returned to its home cage) for several minutes before training resumed. A second pair of errors or balks terminated that day's session. Rats were run until they completed 70 angle trials.

\section{Data Analysis}

The use of path-length titration procedure makes "cumulative path length successfully returned from" a suitable measure of performance. This measure will be used throughout this report for statistical comparisons between actual performance and computer simulation of performance under various strategies ${ }^{1}$ or between the actual performance of different groups. However, because the task becomes more difficult as path length increases (see Results, Figure 3), this measure does not give a clear picture of the acquisition (or lack thereof) of progressively longer paths. Therefore, Figure $2 \mathrm{a}$ has been included as a graphic representation of the mean distance returned from on successful trials (i.e., total path length of successful trials/number of successful trials) in 10-trial blocks (for trial blocks in which there were no successful trials, 0 was substituted for the above ratio). Figure $2 \mathrm{~b}$ represents the proportion of animals, within each group, that reached criterion performance at successive path lengths.

\section{Results}

\section{Pretraining}

Eighty-three percent of the original rats completed pretraining. One or two animals had to be dropped at each stage of pretraining for failure to reach criterion within four to five sessions. The 13 rats that finished the entire study completed each of the pretraining stages in a mean of one or two sessions.

\section{Training}

By the end of 70 trials, the rats were performing successfully from mean RPT paths of $118 \mathrm{~cm}$, that is, they were being released near the center of the room. The mean cumulative distance from which the rats had successfully returned was $40.7 \pm 2.0 \mathrm{~m}$. By comparison, a computer simulation of the performance of 1,300 rats randomly selecting among the eight bottles on each trial and advancing according to the titration rules yielded a mean cumulative distance of $3.9 \pm 1.2 \mathrm{~m}$. The difference between this value and the actual performance is highly significant $(z=30, p<.001)$. The rats' mastery of progressively longer paths is contrasted to the computer simulation of random performance in Figure 2a. Figure $2 b$ compares the proportions of actual and computer-simulated performers reaching criterion at each distance. Another prediction of the randomchoice model is that task difficulty is independent of path length, since the number of choices remains constant. As the trials-to-criterion scores of Figure 3 indicate, however, task difficulty increased with. path length.

An alternative strategy that the rats might have used was to run to the bottle nearest the release point. This strategy would have yielded successful performance at the shorter distances but progressively greater probability of failure at longer distances. ${ }^{1}$

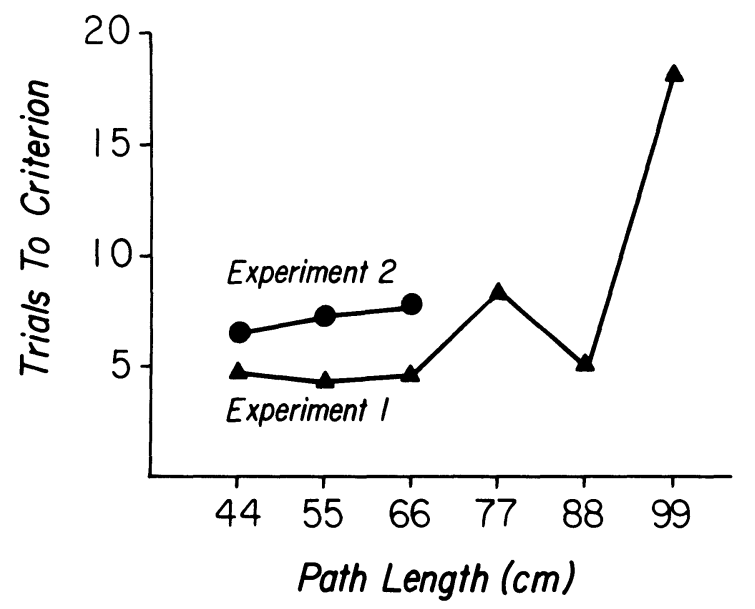

Figure 3. Trials to criterion for successive path lengths. This graph represents only those path lengths at which all subjects reached criterion within 90 trials.

A computer simulation of the performance of 1,300 rats using this strategy produced a mean cumulative distance of $37.7 \pm 3.3 \mathrm{~m}$. This is not significantly different from the actual mean cumulative distance achieved. However, the simulated acquisition curve under the "nearest-bottle strategy" in Figure $2 a$ shows that the average performance under this strategy asymptotes at $99 \mathrm{~cm}$ while the actual rats' mean score in the 60-to-70-trial block is $118 \mathrm{~cm}$ (see the legend of this figure for details of the simulation). Similarly, while $91 \%$ of the actual rats achieved criterion at $110-\mathrm{cm}$ path length, only $31 \%$ of the simulated "nearest-bottle" performers did so (Figure 2b).

The contrast between actual performance and the computer simulations, particularly at greater distances, suggests that the rats were actually using information acquired during the passive transport to determine their choice of bottle upon release. That this information might have been incorporated into some sort of spatial map is indicated by the shape of the rats' return trajectories. While animals sometimes retraced the angle they had been moved through, far more frequently they took "shortcuts" or alternate routes back to the target bottle. The most frequent form of error was "overshooting" the target bottle (see discussion of error analysis in Experiment 3, Figures $1 \mathrm{f}$ and $1 \mathrm{~g}$, and Table 1).

\section{EXPERIMENT 2}

Having successfully developed a procedure for training rats to perform on a RPT task, we turned to the question of the sensory bases of this performance. The similarity of the nearest-bottle strategy simulation to the actual performance at shorter distances suggests that vision might play a role. We therefore enucleated our subjects and attempted to retrain them to RPT. 
Table 1

Experiment 3 Error Analysis

\begin{tabular}{|c|c|c|c|c|c|c|c|c|c|}
\hline & \multicolumn{3}{|c|}{ Error Distance } & \multicolumn{6}{|c|}{ Error Type } \\
\hline & $\begin{array}{l}\text { Under- } \\
\text { shoot }\end{array}$ & $\begin{array}{l}\text { Over- } \\
\text { shoot }\end{array}$ & Other & Straight & $\begin{array}{c}\text { Appropriate } \\
\text { Curvature }\end{array}$ & $\begin{array}{l}\text { Opposite } \\
\text { Curvature }\end{array}$ & $\begin{array}{l}\text { To Experi- } \\
\text { menter }\end{array}$ & Wander & Other \\
\hline Cerebellar & .14 & .73 & .13 & .10 & .21 & .54 & .00 & .14 & .02 \\
\hline Vestibular & .22 & .52 & .26 & .11 & .27 & .34 & .04 & .22 & .03 \\
\hline
\end{tabular}

Note-Error distance: An error arc like that illustrated in Figure $1 \mathrm{~g}$ was drawn on the sketch of the rat's trajectory. An "undershoot" was scored if the intersection of the trajectory and the arc fell in the 45-deg sector on the side of the water bottle stand nearest the release point. An "overshoot" was scored if the intersection occurred in the 45-deg sector on the far side of the stand. Note that an "undershoot" could result from the rat's overestimation of the first leg of the path and/or from underestimation of the second leg; an "overshoot" corresponds to the opposite pattern. Trajectories failing to intersect the error arc within either 45-deg sector were classified as "other." Error type: "Straight" describes a straight-line trajectory that followed the vehicle's long axis. "Appropriate curvature" indicates that the sense of rotation of the rat's trajectory (clockwise or counterclockwise as seen from above) was appropriate to the location of the target bottle. "Opposite curvature" means that the sense of rotation was the opposite to the appropriate one (see Figure 1f). "To experimenter" indicates that the rat came to the experimenter upon release. "Wander" indicates that the rat was picked up $30 \mathrm{sec}$ after release, having neither found the correct bottle nor committed any other error.

\section{Method}

\section{Subjects}

Twenty-four of the 25 rats completing Experiment 1 survived surgery; 18 of these completed Experiment 2. However, data will be reported only from the 13 animals which subsequently also completed Experiment 3.

\section{Enucleation}

Animals were anesthetized with $50 \mathrm{mg} / \mathrm{kg}$ sodium thiopental (Pentathol) ip, their eyeballs excised with fine scissors, and the orbits filled with Bacitracin ointment. A 10-day recovery period elapsed before retraining.

\section{Training}

All animals were retrained in RPT for 90 trials, starting with 44-cm paths and progressing to longer distances following the previous rules.

\section{Results}

By the 70th trial, the rats were successfully returning from a mean path length of $94 \mathrm{~cm}$, which was shorter than 70th trial performance prior to enucleation. On successful trials, the rats once again typically took a curvilinear "shortcut" toward the target until they struck a support arm of the correct stand and followed it to the spout (the analysis of the types of errors and their distributions is deferred until Experiment 3). Actual performance was again compared with a computer simulation of chance performance. However, because the rats were now enucleated, the calculation of chance performance was different from that of Experiment 1. Since rats had only to strike a support arm of the correct stand, the probability of correct responding by chance in this analysis was a function of the angle defined by the release point (as vertex) and the ends of the support arms (see Figure 1f) relative to $360 \mathrm{deg}$. As the path length increased, this angle, and the associated probability of chance success, decreased. The angles formed at each path length were measured on the apparatus and the corresponding ratios entered into the simulation. ${ }^{1}$ The actual mean cumulative distance successfully returned from after enucleation was $34.2 \pm 5.8 \mathrm{~m}$. This was significantly greater than the simulated distance of $9.4 \pm 1.8 \mathrm{~m}(\mathrm{z}=13.8, \mathrm{p}<.001)$.

The postenucleation (Experiment 2) mean cumulative distance was significantly less than the preenucleation (Experiment 1) distance [Wilcoxon test, $T(13)=7, p<.005]$. Similarly, in Figure 2a, the performance curves of Experiment 2 are below those of Experiment 1 and are less steeply accelerated. Figure $2 \mathrm{~b}$ shows that the proportion of rats achieving criterion at successively greater lengths falls off more rapidly in Experiment 2 than in Experiment 1. The trials-to-criterion data of Figure 3 also reflect an increase in task difficulty. Thus, vision can facilitate RPT performance. However, as the above-chance performance of the enucleated animals shows, vision is certainly not necessary for successful RPT performance under these conditions.

\section{EXPERIMENT 3}

We next asked if there was a vestibular contribution to RPT performance by studying the effects of vestibular nuclear lesions on this task. We attempted to restrict the lesions to the rostral end of vestibular nuclear complex from which much of the rostrally directed vestibular projections take origin. The possibility of postlesion motor abnormalities made it necessary to use an additional, "nonvestibular," localization task to evaluate the contribution of such "nonspecific" deficits to any RPT deficit that might appear. An olfactory trail-finding task (OTF) was chosen for this purpose. To make this task as discriminable as possible from the RPT task, we used food instead of water reward. We also realized the necessity for comparing the vestibular lesion with a control lesion and chose, for this purpose, lesions of the cerebellar cortex. Thus, the order of events in Experiment 3 was: (1) all rats trained on OTF, (2) half 
of the rats given vestibular nuclear lesions, half given cerebellar cortex lesions (the groups were equated for prelesion RPT performance levels), (3) all rats retrained on OTF, and (4) all rats retrained on RPT. As an independent evaluation of vestibular function, the rats' air-righting ability was tested following completion of formal training.

\section{Method}

\section{Subjects}

Of the 18 subjects completing Experiment 2, all 6 of the cerebellar-lesioned animals and 7 of the 12 vestibularly damaged animals survived surgery and were able to locomote adequately. During the OTF, the rats were put on a 23-h food-deprivation schedule. They were given five food pellets for $1 \mathrm{~h}$ after the day's testing. Water was available ad lib.

\section{OTF Apparatus}

The Plexiglas training guide was placed on a $1.2 \times 0.9 \mathrm{~m}$ board covered with contact paper. A guillotine door within the guide formed a startbox from which a 1-cm-wide lemon-scented "orienting band" led out of the guide to the choice point at the board's center. Radiating at 70-deg intervals from the choice point were five fixed, unscented, elastic bands, $55 \times 1 \mathrm{~cm}$, and one lemonscented, elastic "trail" band whose far end was movable. The movable end of the trail band could be fixed to any of four possible target positions located midway between the end points of two adjacent unscented bands. Thus, the trail band could form angles of $75,145,215$, and $285 \mathrm{deg}$ with the orienting band. The rat's task after being released from the startbox of the training guide was to follow the ofienting band to the choice point and then find and follow the trail band to its end, where a 0.6-g chocolate chip had been placed.

\section{OTF Procedure}

Pretraining. The animals were initially trained to follow the orienting band to the decision point where the chip had been placed. Then, with only the trail band present in either the 145or 215-deg position, the chip was placed further out along the band in 11-cm increments on successive trials until the rat was following it for the full $55-\mathrm{cm}$ distance. The rats were then additionally pretrained with the trail band in the 75- and 285-deg positions. Finally, they were pretrained with two of the fixed bands present.

Training. After release from the startbox, the rats followed the orienting band to the choice point. They then had to choose the scented band from the five unscented bands and follow it to its end within $30 \mathrm{sec}$ to obtain the chocolate-chip reward. An error was scored and a new trial given if the rat wandered off the board or followed an unscented band for more than $33 \mathrm{~cm}$. Eight trials per session were given; criterion performance was six correct trials in one session.

\section{Surgery}

Aseptic surgery was performed under $50 \mathrm{mg} / \mathrm{kg}$ thiopentol (Pentathol) ip anesthesia. Animals were given bilateral, stereotaxically placed lesions by passing radio-frequency current from a Radionics lesion maker for $10 \mathrm{sec}$ through a coated stainless steel electrode $(0.25 \mathrm{~mm}$ diam, $0.25-\mathrm{mm}$ tip). The experimental lesions were placed in the rostral portion of the vestibular nuclear complex (AP = either lambda $-4.0 \mathrm{~mm}$ or ear bar zero $-2.4 \mathrm{~mm}$, whichever was more anterior. Lateral position $=$ central sinus \pm $1.5 \mathrm{~mm}$. For the DV placement, the electrode was slowly lowered until it touched the floor of the skull, then raised $3.5 \mathrm{~mm}$ ). The control lesions were placed in the cerebellar cortex (AP = lambda $-5.0 \mathrm{~mm} ; \mathrm{ML}=$ central sinus $\pm 1.4 \mathrm{~mm}$. For the DV placement, the electrode was lowered $4 \mathrm{~mm}$, then raised $3 \mathrm{~mm}$ so that it was approximately $1 \mathrm{~mm}^{-}$below the dural surface). During the 14day recovery period, animals were given free access to food and water. Weight was carefully monitored and rats given wet mash as necessary.

\section{Postoperative Testing}

Olfactory trail finding. The animals were initially retrained with only the scented band on the board. Then the five unscented bands were added, and the animals were retested until criterion performance was reached or until 32 trials were given.

RPT testing. As in postenucleation RPT testing, the rats were tested for 90 trials following the error and path-length titration rules, as above.

Air righting. To evaluate vestibular function following the final RPT testing, the animals' air-righting responses were studied. A black cross $(1.75 \times 1.5 \mathrm{~cm})$ was fixed to a diamond-shaped piece of white adhesive tape $(2.0 \times 1.75 \mathrm{~cm})$, which was, in turn, taped to the animal's shaved scalp. Rats were held supine against the underside of a platform elevated $0.5 \mathrm{~m}$ above a table, then released in front of the two hinged mirrors resting on the table. High-speed film (300 frames/sec) were made of their righting movements during the drop. After finding the frame showing first body contact (limb or torso, but not tail) with the table surface, head attitude (pitch and roll) was estimated from the appearance of the black cross in that frame, using a Vanguard motion analyzer (Potegal, Day, \& Abraham, 1977). The pitchand-roll values of three trials per rat were averaged separately.

\section{Results}

\section{Prelesion (Postenucleation) Matching of Groups}

RPT. At 70 trials, the mean cumulative path length successfully returned from by the six rats selected for cerebellar lesions was $32.2 \mathrm{~m}$; for the seven vestibular rats that were eventually capable of being tested postoperatively, it was $35.8 \mathrm{~m}$. The group scores were not significantly different [Mann-Whitney $\mathrm{U}(6,7)=15$, n.s.].

OTF. Eleven of the 13 rats achieved the criterion performance of 6 correct trials in one 8-trial session within a total of 32 trials. The median number of trials to criterion for these 11 rats was 8 . The two animals which failed to achieve criterion had been preassigned to the vestibular group; they were each given a score of 32 (i.e., the maximum number of trials in four sessions). With this score assignment, the groups were not significantly different [MannWhitney $U(6,7)=16$, n.s.].

\section{Postlesion Training}

OTF. The median number of trials to criterion for the cerebellar group was 6; for the vestibular group, it was 7.5. This latter figure includes one rat that had been unable to reach criterion preoperatively but was able to do so postoperatively within 16 trials. While a significant difference does exist between the group scores [Mann-Whitney $\mathrm{U}(6,7)=6$, $\mathrm{p}<.05$ ], the vestibular rats were quickly able to reach the performance level attained by the cerebellar animals.

RPT. The cerebellar group's postlesion perfor- 
mance was identical to its prelesion (postenucleation) performance. By the 70th trial, the cerebellar rats were returning successfully from $91 \mathrm{~cm}$, and by the 90th trial, they were successful from $102 \mathrm{~cm}$ (Figure $2 \mathrm{a}$ ). Their mean pre- and postlesion cumulative path-length scores at 90 trials, $42.4 \pm 7.6 \mathrm{~m}$ and $42.9 \pm 10.6 \mathrm{~m}$, respectively, were not different [Wilcoxon $T(6)=6.5$, n.s.]. By contrast, the vestibular group's performance never approached its prelesion level; by the 70th trial, their performance had reached only $67 \mathrm{~cm}$ (Figure 2a). The difference in vestibular pre- and postlesion cumulative path-length scores at 90 trials, $42.9 \pm 4.4 \mathrm{~m}$ and $30.1 \pm 8.2 \mathrm{~m}$, respectively, was significant [Wilcoxon $T(7)=0, p<.02$ ] The difference between postlesion cerebellar and vestibular performance was significant [MannWhitney $U(6,7)=4, p<.01$ ]. (See also Figure 2b.) The proportion of linear paths successfully returned from by cerebellar rats $(92 \%)$ was significantly greater than the proportion (73\%) of successful linear trials of the vestibular rats [Mann-Whitney $\mathrm{U}(6,7)=5, \mathrm{p}<.02]$. While significantly impaired relative to the cerebellar rats, the vestibular rats were nonetheless better than the simulated chance performance of blind rats.

\section{RPT Error Analysis}

To provide further information about the nature of postlesion performance, we compared and categorized the postoperative errors of both groups. Vestibular animals made a mean of $41.3 \pm 8.7$ errors; cerebellar rats made $33.7 \pm 7.6$. This difference just missed the standard criterion for significance [MannWhitney $U(6,7)=9, p<.051]$. One reason why the difference in overall error rates does not reflect the significant difference in performance between the groups is that the longer path lengths at which the cerebellar rats were performing were more difficult. This inflated their overall error rate.

Errors were classified in Table 1, first, on the basis of distance (undershoot or overshoot, see Figure 1g) and, second, on the basis of qualitative type. There were significant differences in the error $\times$ group interaction in analyses of both error classifications [for distance errors, error $\times$ group interaction $F(2,33)=8.6, p<.01$; for qualitative error classification, error $\times$ group interaction $F(5,66)=4.0$, $\mathrm{p}<.01]$. The cerebellar animals showed pronounced "characteristic" errors. More than half $(\mathbf{7 2 \%})$ of the distance errors of these rats were "overshoots," and more than half $(55 \%)$ of their type errors were "opposite curvature." These two error classifications were also the most frequent direction and type errors, respectively, for the vestibular animals, but accounted for less than $50 \%$ of their errors in both types of analyses. Thus, vestibular error patterns were more variable. Furthermore, the cerebellar rats made half as many errors with appropriate curvature as opposite curvature (see Figure if for definition of curvature). This suggests that, once they had started turning in the appropriate direction, they were better able to localize target-bottle position than were vestibular rats for which turning in the appropriate direction apparently did not give such an advantage. The general conclusion that cerebellar rats knew more than vestibular rats about target location is also supported by the lower frequency of cerebellar "wander" errors.

\section{Air-Righting Response}

The median pitch of the head for the vestibular group was -4 deg; for the cerebellar group, it was $-32 \mathrm{deg}$. The roll median for both groups was -11 deg. However, within the vestibular group, the pitch scores were bimodally distributed. Animals with lesions of the superior vestibular nucleus or bilateral destruction of the anterior portion of the LVN had pitch scores close to or greater than $0 \mathrm{deg}$ $(-4,+7,+8,+55 \mathrm{deg})$; rats with lesions sparing these structures had pitch values close to or less than $-45 \mathrm{deg}(-40,-50,-62 \mathrm{deg})$. It has been shown that pitch values for rats with normal utricular function fall around $-45 \mathrm{deg}$, while values for those with utricular damage fall around or above 0 deg (Potegal et al., 1977; Miller, Barnett, \& Potegal, Note 1). This bimodal distribution was clearly related to the rostral-caudal loci of the lesions and not their sizes.

\section{Neurological Observations}

Immediately postoperatively, all experimental animals showed signs of vestibular damage (Modianos \& Pfaff, 1976; Potegal et al., 1977): head nystagmus, circling, and a rolling, wide-based gait. Three months later, five animals retained milder versions of the gait disorder, and three of these showed mild head nystagmus. Cerebellar animals never showed these deficits.

\section{Lesion Reconstruction}

The rats were overdosed with sodium pentobarbitol and perfused through the heart with $10 \%$ Formalin. The paraffin-embedded brainstems were cut in 10- $\mu$ coronal sections and stained with Luxol fast blue and eosin. Representative sections through the cerebellar and vestibular lesions are shown in Figure 4. Damage to the vestibular nuclei was quantitatively evaluated at four easily identifiable AP levels as follows: (1) Superior (SVN) and medial (MVN) vestibular nuclei at the level at which VIII $\mathrm{N}$. branches can be seen entering the vestibular nuclei (or, if these branches were not visible, the rostralmost section containing the VII $\mathbf{N}$. descending from its genu); (2) SVN, MVN, and lateral (LVN) 
LEVEL 1

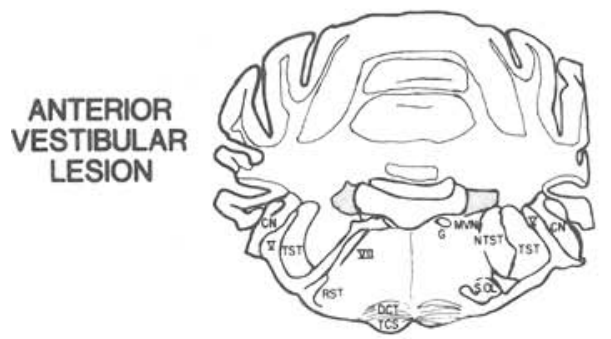

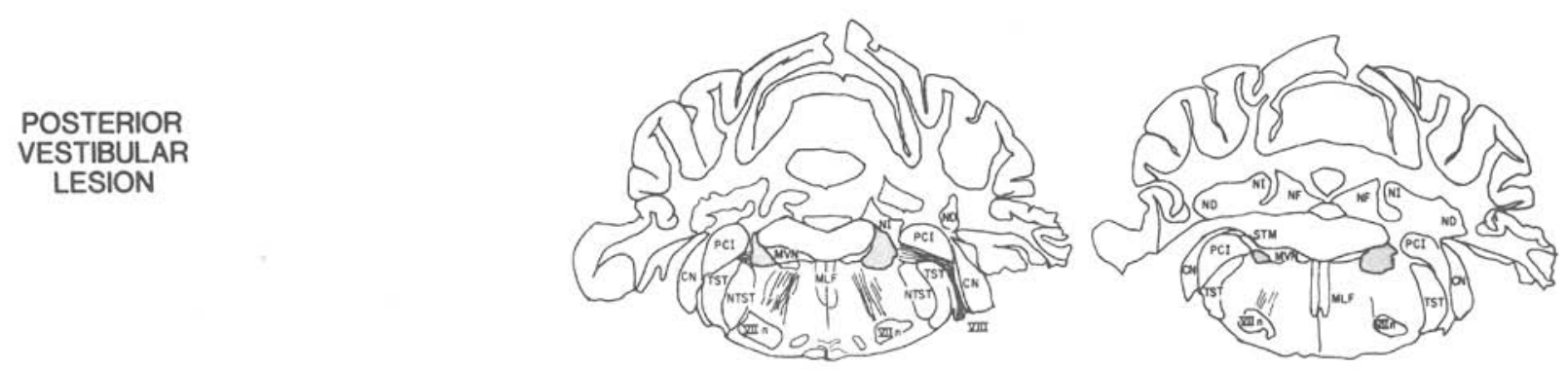
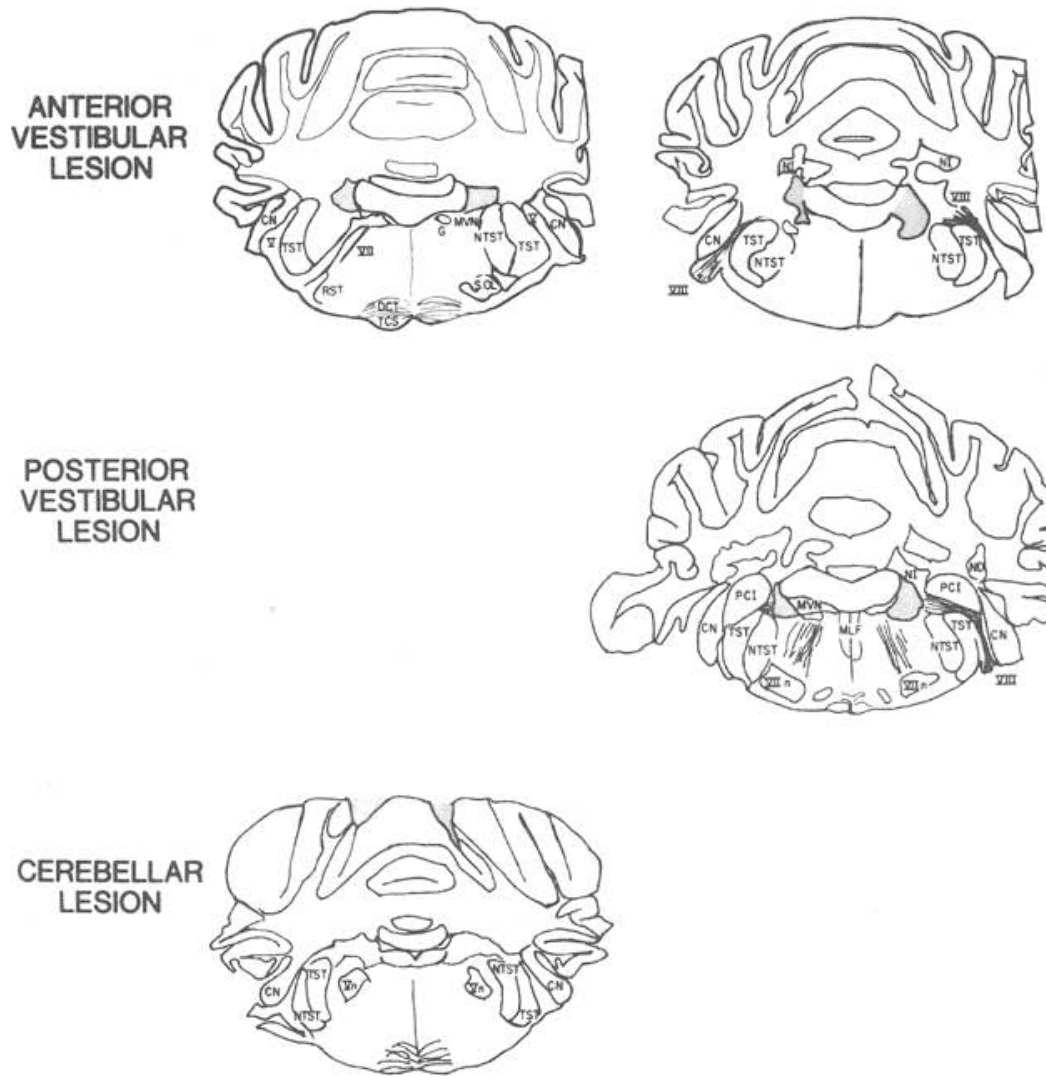

Figure 4. Histological reconstruction of the lesions. See lesion reconstruction section in Experiment 3 for definition of levels. Abbreviation key: $\mathrm{CN}=$ cochlear nucleus, $\mathrm{DCT}=$ decussation of trapezoid corpus, $\mathrm{G}=$ genu of VIIth nerve, MLF $=$ medial longitudinal fasciculus, $\mathrm{MVN}=$ medial vestibular nucleus, $\mathrm{ND}=$ dentate nucleus, $\mathbf{N F}=$ fastigial nucleus, $\mathrm{NI}=$ interstitial nucleus, $\mathrm{NTST}=$ nucleus of the spinal trigeminal tract, $\mathrm{PCI}=$ inferior cerebellar peduncle, $\mathrm{RST}=$ rubrospinal tract, $\mathrm{STM}=$ stria medullaris of the fourth ventricle, $\mathrm{TST}=$ spinal trigeminal tract, $\mathrm{Vn}=$ nucleus of the Vth nerve, $\mathrm{VII}=$ VIIth nerve, VIIn = nucleus of the VIIth nerve, and VIII = VIIIth nerve.

nuclei at a level midway between Levels 1 and 3; (3) MVN and LVN at the level of the appearance of the stria medullaris of the IVth ventricle (i.e., where cerebellum and brainstem separate); and (4) MVN and descending nuclei (DN) at the rostralmost level at which the nucleus solatarius is clearly visible.

Three rats had lesions confined to Levels 1 to 3; four rats had lesions appearing at Levels 2 to 4 . In the anterior group, at Level 1 all subjects suffered $50 \%$ or greater bilateral destruction of the SVN and MVN and, at Level 2, 50\% or greater bilateral LVN destruction. The posterior group at Level 2 suffered either complete bilateral destruction of LVN $(\mathrm{N}=2)$ or partial LVN damage combined with complete unilateral destruction of MVN and variable damage to the contralateral MVN $(\mathbf{N}=2)$. Variable damage to MVN, LVN, and DN occurred at Levels 3 and 4 in the posterior group.

\section{DISCUSSION}

Beritoff has reported that animals of several species can successfully return to a starting point after being passively transported away from it. Using the procedures reported in this paper, we have extended the demonstration of reliable performance on this task to rats. Most of their correct responses did not involve retracing the right-angle paths through which the animals were transported; instead, they generally took a "shortcut" to the target bottle. This suggests the possibility that the rats were incorporating the information acquired during passive transport into some more general spatial map of their environment.

We also found that enucleation retarded, but did not preclude, successful RPT performance. Thus, vision is not necessary for RPT performance. This result is consistent both with Beritoff's claims and 
some of our earlier, preliminary observations (Miller et al., 1978). Even when blinded, the rats took shortcuts back to the target bottles.

In contrast to enucleation, vestibular nuclear damage profoundly impaired rats' abilities to perform at the longer path lengths. This deficit was not due to nonspecific brain damage, since cerebellar cortex lesions had no such effects. Neither can the RPT deficit be explained solely in terms of motor difficulties. Rats were retrained on OTF immediately postoperatively, when the movement disorders were most serious. They were successful in relearning this task. Indeed, one of the movement-impaired vestibular animals which had not learned OTF during initial training did learn it postoperatively. RPT training began after OTF retraining, when motor impairments had grown less severe, yet the subjects showed a profound RPT deficit. Additionally, rank ordering of rats by motor impairment did not correlate with performance in either task. Furthermore, the deficit cannot be explained as a completely generalized incapacity in spatial problem solving, since the animals with vestibular lesions had only a mild and transient deficit on OTF. Of course, while OTF and RPT are similar in demanding target localization using nonvisual cues, they differ along a number of dimensions, including the nature of the reward, and so on.

Thus, we have developed a spatial orientation task in which the usual kinesthetic, proprioceptive, and visual inputs during active locomotion have been precluded and found unnecessary for successful performance. On the other hand, the integrity of the vestibular system is necessary for optimal performance. What specific role might vestibular input be playing? The simplest process, consistent with the impairment on linear trials, is that the vestibular system is used in its "direction orientation" mode to keep the animal "on track" toward its intended location during its return journey. In a second, more complex process, the vestibular information generated during passive transport might be used by the rats in calculating their return trajectory perhaps by being incorporated into some kind of spatial map. The right-angle turn would presumably be the most salient aspect of such information, although the accelerations at the beginning and end of each leg of the angle might also be used. It is difficult to distinguish these two processes (or their combined effect) using blinded rats as we did in this study. However, Miller, Potegal, and Abraham (1981) have recently found that vestibular nuclear lesions also impair RPT performance in visually intact rats. Since, in these animals, keeping "on track" toward an intended target location can be done visually, it is more likely that a vestibular spatial mapping function is also involved in our task.
Under either hypothesis, it is reasonable to suppose that the vestibular input must be processed at higher levels of the nervous system concerned with navigation. One of the functions of the vestibular pathways ascending to the forebrain may be the transfer of just such information. One of the structures receiving this input is the caudoputamen (e.g., Potegal, Copack, de Jong, Krauthamer, \& Gilman, 1971), a structure which has also been implicated in spatial orientation (Potegal, 1982). The following paper by Abraham, Potegal, and Miller (1983) is an exploration of the role of the caudoputamen in a RPT task.

Two additional points should be made. While the integrity of the vestibular system is necessary for optimal performance in RPT, rats with vestibular nuclear damage could still perform at above-chance levels. This may mean either that the lesions did not eliminate all vestibular input and/or that somatic sensory stimulation occurring during passive transport (i.e., pressure changes and contact with the walls of the vehicle) provides information for subsequent target localization. These factors may also account for the overall similarity in the error patterns of vestibular and cerebellar subjects. However, to the extent which RPT-relevant somatosensory information is processed by the dorsal column nuclei, the latter hypothesis is rendered less likely by recent data indicating that animals with dorsal column lesions successfully perform RPT (Miller et al., 1981). It should also be pointed out that our task is somewhat unusual in that the rats were being transported backward. This was done because shaping to the task was made easier. While this does not vitiate any of our conclusions, generalizations to other situations can only be made with caution. In particular, the question of whether vestibular information is used for spatial guidance in other, less restricted, conditions remains to be investigated.

\section{REFERENCE NOTE}

1. Miller, S., Barnett, B., \& Potegal, M. Cues for path-finding with passive movement exposure to path. Paper presented at the 49th annual meeting of the Eastern Psychological Association, Washington, D.C., April 1978.

\section{REFERENCES}

Abraham, L., Potegal, M., \& Miller, S. Evidence for caudate nucleus involvement in an egocentric spatial task: Return from passive transport. Physiological Psychology, 1983, 11, 11-17.

BARLow, J. S. Inertial navigation as a basis for animal navigation. Journal of Theoretical Biology, 1964, 6, 76-117.

BERITOFF, J. S. Neural mechanisms of higher vertebrate behavior (W. T. Liberson, trans.). Boston: Little, Brown, 1965.

DASHiEll, J. F. Direction orientation in maze running by the white rat. Comparative Psychology Monographs, 1930, 7(2, Whole No. 32).

DAshiell, J. F. The role of vision in the spatial orientation of 
the white rat. Journal of Comparative Physiological Psychology, 1959, 52, 522-526.

ExNER, S. Negative Versuchsergebnisse das Orientierungsvermogen der Brieftauben. Sitzung-Berichte der Akademie der Wissenschaften in Wien, 1893, 102, 318-331.

Honzik, C. H. Maze learning in rats in the absence of specific intra- and extra-maze stimuli. University of California Publications in Psychology, 1933, 6, 99-144.

Jones, M., \& Milsum, J. H. Characteristics of neural transmission from the semicircular canal to the vestibular nuclei of cats. Journal of Physiology, 1970, 209, 295-316.

Miller, S., Potegal, M., \& Abraham, L. Vestibular involvement in spatial orientation. Society for Neuroscience $A b$ stracts, 1981, 7, 484.

Modianos, D. T., \& Pfaff, D. W. Brainstem and cerebellar lesions in female rats. I. Tests of posture and movement. Brain Research, 1976, 106, 31-46.

Potegal, M. Vestibular and neostriatal contributions to spatial orientation. In M. Potegal (Ed.), Spatial abilities: Development and physiological foundations. New York: Academic Press, 1982.

Potegal, M., Day, M. J., \& Abraham, L. Maze orientation, visual and vestibular cues in two-maze spontaneous alternation of rats. Physiological Psychology, 1977, 5, 414-420.

Potegal, M., Copack, P., deJong, J. M. B., Krauthamer, G., \& Gilman, S. Vestibular input to the caudate nucleus. Experimental Neurology, 1971, 32, 448-465.

RiLey, D. A., \& Rosenzweig, M. R. Echolocation in rats. Journal of Comparative Physiological Psychology, 1957, 50, 323-328.

Walcott, C., Gould, J. L., \& Kirschvink, J. L. Pigeons have magnets. Science, 1979, 205, 1027-1028.

WAtson, J. D. Kinesthetic and organic sensations: Their role in the reaction of the white rat to the maze. Psychological Review Monographs, 1907, 8(Whole No. 33).

WolfLe, D. L. The effect of continuous interchange of alley sections on the maze behavior of rats. Journal of Comparative Physiological Psychology, 1935, 9, 91-106.

ZoladeK, L., \& Roberts, W. A. The sensory basis of spatial memory in the rat. Animal Learning \& Behavior, 1978, 6, 77-81.

\section{NOTE}

1. Three simulations using 10 independent multiplicant randomnumber generators (IBM SSB RANDU) were programmed and run on an IBM 4331. A run began with a starting distance of 44, successive trials were determined as successes or failures according to one of the three probability rules listed below, but the "achieved distance" was incremented, decremented, or left unchanged according to the running rules (see Methods, Experiment 1). For each simulation, 1,300 runs were made. The percentage of subjects reaching criterion at each path length was calculated, as was the mean path length successfully returned from in 10 trial blocks.

To compute the success probability values for the "nearestbottle" strategy, each of the four possible paths for the corner bottles and two paths for midwall bottles were laid out for each path length (e.g., Figures 1d, 1e). The proportion of the six possible paths for which the correct target bottle happened to be the one nearest the release point was determined. To compute the chance performance success angle for Experiments 2 and 3, the actual paths at each length were laid out on the floor of the testing room and the mean chance success angle was found for all of the path configurations of a given length.

Success Probability Values for Simulations

\begin{tabular}{|c|c|c|c|c|c|c|c|c|c|c|}
\hline \multirow{2}{*}{$\begin{array}{l}\text { Exper- } \\
\text { iment }\end{array}$} & \multirow{2}{*}{$\begin{array}{l}\text { Strategy Being } \\
\text { Simulated }\end{array}$} & \multicolumn{9}{|c|}{ Path Length (in Centimeters) } \\
\hline & & 44 & 55 & 66 & 77 & 88 & 99 & 110 & 121 & 132 \\
\hline 1 & Chance Performance* & .125 & .125 & .125 & .125 & .125 & .125 & .125 & .125 & .125 \\
\hline 1 & Nearest Bottle Strategy & 1.00 & 1.00 & 1.00 & 1.00 & .67 & .67 & .33 & .33 & .000 \\
\hline 2,3 & Chance Performance $\dagger$ & .27 & .19 & .14 & .13 & .11 & .10 & .09 & .08 & .07 \\
\hline
\end{tabular}

*Random choice of one of eight. $\quad$ tSuccess angle/360 deg.

(Manuscript received November 8, 1982;

revision accepted for publication February 14, 1983.) 